\title{
Departamentalization in the Federal Universities of the Northeast: Isomorphism or new settings?
}

\section{Patrícia de Carvalho Marques ${ }^{1}$, José Luiz de Moreira Carvalho²}

\author{
${ }^{1,2}$ Universidade Federal do Vale do São Francisco (UNIVASF).
}

Email: paty-cmarques94@live.com,jose.carvalho@univasf.edu.br

Received: March 17th, 2017

Accepted: April $28^{\text {th }}, 2017$

Published: June $30^{\text {th }}, 2017$

Copyright $(02016$ by authors and Institute of Technology Galileo of Amazon (ITEGAM) This work is licensed under the Creative Commons Attribution International License (CC BY 4.0).

http://creativecommons.org/lic enses/by/4.0/

\begin{abstract}
The beginning of the XXI Century represented a period of expansion of the Brazilian Federal Universities, which also represented a period of creation of new universities. The creation of a new university represents an opportunity for innovation in its organization, but "isomorphic forces" can act to repeat preexisting models. Therefore, the objective of this article is to analyze how the federal universities of the Northeast region are structured, at their strategic level of decision (Pro-Reitories) and to make a comparative study between the most traditional and the most recent universities. Through a descriptive and documental research, done in a multi-case study, it was sought to know better how they are departmentalized and how much this implied or not in innovations in this structure.
\end{abstract}

Keywords: Departmentalization, Universities, Pro-Rectorates.

\section{Departamentalização nas universidades federais do nordeste: is omorfismo ou novas configurações?}

\section{RESUMO}

O início do Século XXI representou um período de expansão das universidades federais brasileiras, o que representou também um período de criação de novas universidades. A criação de uma nova universidade representa uma oportunidade à inovação na sua organização, mas "forças isomórficas" podem atuar no sentido de repetir modelos pré-existentes. Portanto, o objetivo deste artigo é analis ar como estão estruturadas as universidades federais da região Nordeste, no seu nível estratégico de decisão (Pró-Reitorias) e fazer um estudo comparativo entre as universidades mais tradicionais e as mais recentes. Através de uma pesquisa descritiva e documental, feita em um estudo multi-caso, buscou-se conhecer melhor como as mesmas estão departamentalizadas e o quanto is so implicou ou não em inovações nessa estrutura.

Palavras Chaves: Departamentalização, Universidades, Pró-Reitorias.

\section{INTRODUÇÃO}

As universidades federais brasileiras passaram por um período de expansão nos últimos anos. Segundo informações do Ministério da Educação [1], com a criação do Plano Nacional da Educação (PNE), em 2001, foram estabelecidos por 10 anos, os programas de expansão do ensino superior federal. Sua primeira fase compreendeu o período de 2003 a 2007 e teve como meta principal interiorizar o ensino superior público federal, o qual contava até o ano de 2002 com 45 universidades federais e 148 campus/unidades. Entre 2003 e 2010, houve um aumento de 45 para 59 universidades federais e de 148 para 274 campus. Com a interiorização, também se elevou o número de municípios atendidos por universidades federais de 114 para 272 [1].

Transformações importantes nas Instituições Federais de Ensino Superior (IFES) também aconteceram por conta da Lei $\mathrm{N}^{\circ}$ 12.711, de 29 de agosto de 2012 [2], que estabeleceu mecanismos de acesso através de cotas raciais, sociais e para alunos de escolas públicas. E também por conta do Programa Nacional de Assistência Estudantil (PNAES), que tem como objetivo promover a permanência de estudantes em situação de vulnerabilidade social [3]. 
Por tudo isso, o objetivo deste artigo é analisar como estão estruturadas as universidades federais da região Nordeste, no seu nível estratégico de decisão (Pró-Reitorias) e fazer um estudo comparativo entre as universidades mais tradicionais e as criadas no Século XXI. Através de uma pesquisa descritiva e documental, feita em um estudo multi-caso focando as universidades federais da região Nordeste, busca-se conhecer melhor como as mesmas estão departamentalizadas e o quanto o surgimento de novas universidades representaram ou não inovações nessa estrutura. Nesse sentido, destaca-se também a questão do isomorfismo. Os autores agradecem à Fundação de Amparo ao Pesquisador do Estado da Bahia (FAPESB) pelo apoio.

\section{MÉTODOLOGIA APLICADA}

O presente estudo consiste em uma pesquis a descritiva e documental, feita em um estudo multi-caso. Conforme [4], a pesquisa documental caracteriza-se pela restrição da fonte de coleta de dados, que se dá por documentos, escritos ou não, constituindo as fontes primárias, podendo ser realizada no momento em que o fato ou fenômeno ocorre, ou depois.

Para [5], a pesquisa documental é muito parecida com a bibliográfica, diferindo na natureza das fontes, pois esta forma vale-se de materiais que não receberam ainda um tratamento analítico, ou que ainda podem ser reelaboradas de acordo com os objetos da pesquisa.

A análise documental, segundo [6], constitui uma técnica importante na pesquisa qualitativa, seja complementando informações obtidas por outras técnicas, s eja desvelando as pectos novos de um tema ou problema.

Por sua vez, conforme [7], a pesquisa descritiva envolve o estudo, análise, registro e interpretação dos fatos do mundo físico sem a interferência do pesquisador. Para [5], esse tipo de pesquis a visa descrever as características de determinadas populações ou fenômenos, estando uma de suas peculiaridades na utilização de técnicas padronizadas de coleta de dados, tais como o questionário e a observação sistemática.

Já o estudo de caso, de acordo com [8] pode ser restrito a uma ou a várias unidades, caracterizando-o como único ou múltiplo. Para [9], nos estudos de multi-casos o pesquisador pode ter a possibilidade de estudar dois ou mais sujeitos, organizações, entre outros. Esses estudos vis am analis ar um objeto de estudo de maneira singular, mesmo que, posteriormente, tenham sido observadas semelhanças com outros casos, retratando a realidade de forma completa e profunda. O estudo de multi-casos tem se mostrado conveniente na identificação de três fatores: fatores comuns a todos os casos no grupo escolhido; fatores não-comuns a todos, mas apenas a alguns subgrupos; fatores únicos em caso específico.

Considerando o embasamento teórico sobre a departamentalização, através de uma pesquisa documental nas páginas das universidades na internet, buscou-se conhecer como as mesmas estão organizadas no nível estratégico das PróReitorias e investigou-se o aspecto do isomorfismo e a influência das novas políticas governamentais nessas estruturas. As decisões de nível estratégico das organizações, que compreendem as grandes escolhas de objetivos organizacionais e meios para realizá-los geralmente são tomadas no nível hierárquico mais alto, já que afetam a organização inteira. A alta administração, embora sendo a res ponsávelfinal pelas decisões estratégicas pode recorrer a funcionários de outros níveis, como fonte de informação ou como participantes ativos do processo [10].

\section{REFERENCIAL TEÓRICO}

\section{III.1 O ISOMORFISMO}

De acordo com [11], o isomorfismo pode ser identificado segundo duas vertentes distintas: o competitivo, que considera uma racionalidade que dá ênfase ao mercado de competição, e o institucional, relacionado a uma ênfase estrutural vinculada ao escopo burocrático.

No isomorfismo institucional, os mesmos autores descrevem três tipos: (1) coercitivo, como resultado de pressões formais e informais, exercidas por outras organizações das quais as organizações dependem; (2) mimético, onde as organizações se modelam, adotando as formas das outras organizações do meio, por intermédio da transferência ou rotatividade de funcionários, e (3) normativo, em decorrência principalmente da profissionalização. Com isso, considera-se que, a partir da ideia de legitimação, as ações das organizações tornam-se homogêneas através de mecanismos isomórficos em relação ao ambiente institucional.

As organizações de um mesmo ambiente institucional têm tendência de apresentarem similaridades estruturais e de processo, pela possível comparação via profissionalização dos seus atores, como uma das causas, pois são as pessoas atuando no campo organizacional quem define as estruturas segundo um conjunto de crenças internalizadas a partir dos valores compartilhados no contexto. $\mathrm{O}$ isomorfismo, então, atua como um processo que limita a diferenciação, obrigando a unidade a se as semelhar com o coletivo de seu campo [12][13].

Por tudo isso, ainda que a criação de uma nova universidade representasse uma oportunidade à inovação, "forças isomórficas" podem atuar no sentido contrário, o que a levaria a repetir modelos pré-existentes em universidades tradicionais.

\section{III.2 A DEPARTAMENTALIZAÇÃO}

A departamentalização, também conhecida, segundo [14], como especialização horizontal, corresponde ao aperfeiçoamento de atividade e de conhecimentos, caracterizando-se sempre pelo crescimento horizontal do organograma. Um departamento é uma unidade de trabalho responsável por uma função ou por um conjunto de funções. Logo, para [10], a distribuição das funções entre os departamentos depende de diversos fatores, como o tamanho da organização e a disponibilidade de recursos.

Conforme [14] a especialização horizontal deve ser regida pela homogeneidade, cujo principal conceito é a designação de funções em unidades organizacionais por semelhança de conteúdo, tendo como grande objetivo alcançar operações mais eficientes e econômicas. Desta forma, para [15] a departamentalização é agrupada segundo um critério de semelhança de atividades e correspondentes recursos (humanos, financeiros, materiais e equipamento) em determinadas unidades. Seguindo o princípio citado, os gestores poderão organizar melhor as atividades sob sua responsabilidade quando es tiverem agrupados de alguma forma lógica. 
Logo, a departamentalização envolve o processo de determinar unidades compostas de grupos com cargos relacionados; À medida que a organização cresce, a dimensão horizontal amplia automaticamente pelo surgimento de novas unidades, e envolve ainda a descentralização, na qual as decisões devem ser tomadas pelos que detêm as informações mais importantes, não devendo se restringir ao nível mais alto, com is so há a maior participação no processo de tomada de decisão, o que eleva a moral e a motivação em todos os níveis [16].

\section{III.3 OS TIPOS DE DEPARTAMENTALIZAÇÃO}

Para [16], as técnicas de departamentalização variarão dependendo da organização e da época. Elas dependerão da tecnologia, da filosofia administrativa, do capital, da força de trabalho, do tamanho e de uma variedade de outros fatores. A seguir são apresentadas as formas de departamentalização das atividades de uma organização com seus aspectos principais:

a) Departamentalização por Quantidade: Para uma empresa trabalhar com a departamentalização por quantidade, segundo [15] ela deve agrupar certo número de pessoas não diferenciáveis que a partir dessa situação têm por obrigação executar tarefas sob as ordens de um superior. Para [17], esse tipo de departamentalização tem por objetivo dividir a organização pela quantidade de pessoas existente em cada conjunto.

\section{b) Departamentalização}

Funcional:

A departamentalização funcional (ou por funções) tem como principal característica, segundo [18], agrupar funções comuns ou atividades semelhantes para formar uma unidade organizacional. Este tipo de departamentalização ocorre quando se reúne pessoas em um mesmo trabalho, em um mesmo processo, para mesma clientela, em um mesmo lugar. Ademais, para [14] e [15] consiste no agrupamento das atividades e tarefas de acordo com as funções principais desenvolvidas dentro da organização. Éainda o critério mais utilizado para organizar atividades empresariais.

c) Departamentalização Territorial: A departamentalização territorial, que também pode ser chamada de regional, por área ou geográfica é designada por [18] como o agrupamento de atividades de acordo com os lugares onde são localizadas as operações. Para [15] esse tipo de especialização horizontal se baseia no princípio de que todas as atividades que se realizam em determinado território devem ser agrupadas e colocadas sob as ordens de um executivo. Segundo [14] é utilizada por organizações que cobrem grandes áreas geográficas e cujos mercados são extensos, como por exemplo, as empresas multinacionais.

d) Departamentalização por Produtos ou Serviços: A departamentalização por produto foi criada para transformar a organização em tamanho menor e mais rápida nas decisões, já que, para [18], quando é preciso tomar decisões rápidas, a abordagem funcional deixa a desejar. Conforme [14] a mesma tem como características a diferenciação e o agrupamento de atividades de acordo com o produto ou serviço realizado, ou seja, todas as atividades requeridas para suprir um produto ou serviço deverão ser agrupadas no mesmo departamento. O mesmo autor cita como exemplo uma instituição de ensino, que pode departamentalizar suas atividades em curso fundamental, curso de secretariado, cursos de aperfeiçoamento técnico, etc.

e) Departamentalização por Clientela: A departamentalização por clientela (ou por cliente) consiste em agrupar atividades de maneira que focalizem o uso determinado de um produto ou serviço, este método para [18] é utilizado no agrupamento de vendas ou serviços. Além disso, este tipo de departamentalização predomina, segundo [16], quando maior ênfase é dada no atendimento ao cliente. Para [14] essa organização envolve a diferenciação e o agrupamento das atividades conforme o tipo de pessoa para quem o trabalho é executado. O que constitui a base para este tipo de departamentalização são as características dos clientes.

\section{f) Departamentalização por Processo: A} departamentalização por processo, para [18], consiste no agrupamento de atividades que focalizam equipamento ou processo de produção. Por exemplo, as atividades de uma fábrica podem ser agrupadas em: perfurar, esmerilhar, soldar, montar e fazer o acabamento. Tal processo é definido, segundo [14] como um conjunto de atividades estruturadas e destinadas a resultar um produto especificado para um determinado cliente ou mercado. É utilizado com mais frequência nas empresas industriais nos níveis mais baixos da estrutura organizacional (produção).

g) Departamentalização por Projetos: A departamentalização por projetos é uma estratégia utilizada em empresas de grande porte e que, de acordo com [14] produzem produtos que envolvem grande concentração de recursos e prolongado tempo para produção. Para [15] baseia-se na definição de projeto, que é um trabalho com datas de início e término, com resultado final previamente estabelecido, em que são alocados e administrados os recursos, tudo is so sob a responsabilidade de um coordenador.

h) Departamentalização Matricial: Do ponto de vista evolutivo, para [15] a departamentalização matricial surgiu por que as formas tradicionais de organizar não eram eficazes para lidar com atividades complexas, envolvendo várias áreas do conhecimento científico e com prazos determinados para a sua realização. Esta especialização horizontal, segundo [18], vem da união de um ou mais tipos de departamentalização. Um exemplo é o da estrutura funcional com a estrutura de projetos. Em consequência disso, pode haver um conflito interno, que pode ser evitado caso exista clara definição de atribuições de cada um dos elementos da estrutura organizacional.

i) Departamentalização Mista: A departamentalização mista é a que ocorre com mais frequência nas organizações, já que cada parte da empresa, segundo [15] deve ter uma estrutura que mais se adapte à sua realidade organizacional. Para [17], is so ocorre por ser muito difícil que as organizações grandes e complexas utilizem uma só técnica de departamentalização. Sendo assim, a estrutura organizacional dessas empresas é uma mistura das várias formas já apresentadas. Pode haver conflitos internos pela inovação e disputas de poder; conflitos internos pela falta de definição de atribuições e antipatias pessoais; e duplicação de esforços em trabalhos iguais, mas em áreas diferentes. 


\section{RESULTADOS E DISCUSSÕES}

Como já explicado, a partir de uma pesquisa descritiva e documental, pesquisou-se as formas de departamentalização utilizadas pelas universidades federais do Nordeste. Ao todo, são 18 universidades, sendo que 7 delas
(UFSB, UFOB, UFRB, UNIVASF, UFERSA, UFCA e UNILAB) são novas e as outras 11 são mais antigas.

Os Quadros 1, 2 e 3 mostram uma síntese dos resultados encontrados. Procurou-se agrupar de acordo com a denominação, mesmo com as pequenas diferenças que pode haver entre elas. Na primeira tabela estão enfocadas as atividades de Ensino, Pesquisa e Extensão.

Quadro 1: Ensino, Pesquisa e Extensão nas universidades federais do Nordeste.

\begin{tabular}{|c|c|c|c|c|c|c|c|c|c|c|c|c|c|c|c|c|c|c|}
\hline UNIVERSIDADES / PRÓ-REITORIAS & UFBA & UFS & UFAL & UFPE & UFRPE & UFPB & UFCG & UFRN & UFC & UFPI & UFMA & UFSB & UFOB & UFRB & UNIVASF & UFERSA & $\mathrm{UFCA}$ & UNLAB \\
\hline \multicolumn{19}{|l|}{ EISINO/GRADDACÃO } \\
\hline \multicolumn{19}{|l|}{ Gestão Acadèmica } \\
\hline \multicolumn{19}{|l|}{ Graduacào e Acōes Afirmativas } \\
\hline \multicolumn{19}{|l|}{\begin{tabular}{|l|l|l|l|} 
PÓS-GRADDAĊOO \\
\end{tabular}} \\
\hline \multicolumn{19}{|l|}{\begin{tabular}{|l|} 
Pesquisa e Pós-Graduaçó \\
\end{tabular}} \\
\hline \multicolumn{19}{|l|}{ Pesquisa, Pós-Graduaço e Inovaçăo } \\
\hline \multicolumn{19}{|l|}{ Pesquisa, Pós-Graduacào, Criaçò e Inovaçä } \\
\hline \multicolumn{19}{|l|}{ PESQUTSA } \\
\hline \multicolumn{19}{|l|}{ Pesquisa, Criaģà e Inovaçä } \\
\hline \multicolumn{19}{|l|}{ Pesquisa e Extensäo } \\
\hline \multicolumn{19}{|l|}{ EXIENSÃOO } \\
\hline \multicolumn{19}{|l|}{ Extensão e Cultura } \\
\hline \multicolumn{19}{|l|}{ Extensäo, Arte e Cultura } \\
\hline \multicolumn{19}{|l|}{ Extensão e Assuntos Comunitários } \\
\hline Extensà, Cultura e Empreendedorismo & & & & & & & & & & & & & & & & & & \\
\hline
\end{tabular}

Fonte: Autores, (2106).

Ensino de Graduação:

De acordo com o quadro 1, na maioria das universidades federais nordestinas existe uma Pró-Reitoria Pós-Graduação.

Em 5 casos a Pró-Reitoria é exclusiva para a PósGraduação. Em outras 7, Pesquis a e Pós-Graduação estão juntas e em 4 coexistem Pesquisa, Pós-Graduação e Inovação. Uma configuração diferente ocorre na UFRB, com a Pró-Reitoria de Pesquisa, Pós-Graduação, Criação e Inovação. Na UFSB não foi encontrado nenhum setor que trate da Pós-Graduação.

\section{$\checkmark \quad$ Pesquisa}

Como visto anteriormente, as atividades de pesquisa estão junto com a Pós-Graduação (e Inovação) em 12 das 18 universidades pesquisadas. Uma Pró-Reitoria exclusiva para a Pesquisa ocorre em 2 casos (UFRN, UFPI). Na UFBA, Pesquisa está junto com Criação e Inovação e na UFCG, junto com a específica para Ensino/Graduação, com essa nomenclatura. Exceções são a UFPE e a UFSB, que usam o termo "Gestão Acadêmica". Uma configuração diferente ocorre na UFOB, onde há a Pró-Reitoria de Graduação e Ações Afirmativas.

Extensão. A UFSB não tem Pró-Reitoria com nomenclatura relacionada à Pesquisa.

\section{$\checkmark \quad$ Extensão}

No caso das atividades de Extensão, há também formas diversas de departamentalização. Na UFCG, já citada, há a PróReitoria de Pesquisa e Extensão. Em 9 universidades, é exclusiva para a Extensão. Já na UNILAB, UFPE, UFOB e UFERSA, Arte e Cultura estão juntas com a Extensão. UFPB e UFS tem PróReitoria de Extensão e Assuntos Comunitários. E na UFMA há a Pró-Reitoria de Extensão, Cultura e Empreendedorismo. Outro conjunto de atividades exercido pelas pró-reitorias envolve a área de gestão das universidades. Como visto no quadro 2, há uma diversidade de formas de departamentalizar essas funções. 
Quadro 2: Atividades administrativas nas universidades federais do Nordeste.

\begin{tabular}{|c|c|c|c|c|c|c|c|c|c|c|c|c|c|c|c|c|c|c|}
\hline \multirow{2}{*}{\begin{tabular}{|l|} 
UNIVERSIDADES / PRO-REITORIAS \\
ADDINISTRAÇC̃ O, PLANEJAIIENTO E \\
ORÇAIIENTO \\
\end{tabular}} & UFBA & UFS & UFAL & UFPE & UFRPE & UFPB & UFCG & UFRN & $\mathrm{UFC}$ & UFPI & UFMA & UFSB & UFOB & UFRB & UNIVASF & UFERSA & UFCA & UNILAB \\
\hline & & & & & & & & & & & & & & & & & & \\
\hline Administraçào & & & & & & & & & & & & & & & & & & \\
\hline Administração e Infra-estrutura & & & & & & & & & & & & & & & & & & \\
\hline Gestão e Administração Financeira & & & & & & & & & & & & & & & & & & \\
\hline Gestão e Orçamento & & & & & & & & & & & & & & & & & & \\
\hline Gestão Instituciona & & & & & & & & & & & & & & & & & & \\
\hline Planejamento & & & & & & & & & & & & & & & & & & \\
\hline Planejamento e Administraçà & & & & & & & & & & & & & & & & & & \\
\hline Planejamento e Coordenação Geral & & & & & & & & & & & & & & & & & & \\
\hline Planejamento e Desenvolvimento Institucional & & & & & & & & & & & & & & & & & & \\
\hline Planejamento, Orcamento e Financas & & & & & & & & & & & & & & & & & & \\
\hline GESTÃO DE PESSOAS & & & & & & & & & & & & & & & & & & \\
\hline Gestào de Pessoas e Qualidade de Vida & & & & & & & & & & & & & & & & & & \\
\hline Desenvolvimento de Pessoas & & & & & & & & & & & & & & & & & & \\
\hline Recursos Humanos & & & & & & & & & & & & & & & & & & \\
\hline
\end{tabular}

Fonte: Autores, (2106).

$\checkmark$ Administração, Planejamento e Orçamento

Na gestão das universidades, há, claramente, uma divisão das funções de Administração (existente em 11 casos) e Planejamento (8 casos). Há algumas variações, como Administração e Infra-estrutura (UFOB), Gestão e Orçamento (UNIVASF), Planejamento e Coordenação Geral (UFRN), Planejamento e Desenvolvimento Institucional (UNIVASF, UFOB) e Planejamento, Orçamento e Finanças (UFBA, UFPE, UFCA). Na maioria dos casos, há duas pró-reitorias dedicadas às atividades administrativas. As exceções são as de Gestão e Administração Financeira (UFCG, UFMA), Gestão Institucional (UFAL), Planejamento e Administração (UFSB) e Planejamento (UFRPE), que concentram numa só todas as atividades.

\section{$\checkmark$ Gestão de Pessoas/RH}

A área de RH/Gestão de Pessoas tem status de PróReitoria em 12 universidades, sob diversas denominações (Gestão de Pessoas e do Trabalho, Gestão de Pessoas e Qualidade de Vida, Desenvolvimento de Pessoas, Recursos Humanos). Já em 6 casos essa área não tem esse status, ficando vinculada a outra ou então tendo uma forma diferente de departamentalização. Na UNILAB não existe uma Pró-Reitoria tratando deste assunto, mas há uma coordenação de Gestão de Pessoas vinculada à PróReitoria de Administração. Já na UFSB uma Diretoria de Gestão de Pessoas vinculada à Pró-Reitoria de Planejamento e Administração. Na UFCG há uma Secretaria de Recursos Humanos que trata do as sunto desta área. Na UFPI, na UFRPE e na UNIVASF existe uma Superintendência de Gestão de Pessoas. Por fim, no Quadro 3 estão as demais pró-reitorias, com destaque para as temáticas de assistência estudantil, ações afirmativas, tecnologia de informação.

Quadro 3: Outras Pró-Reitorias.

\begin{tabular}{|c|c|c|c|c|c|c|c|c|c|c|c|c|c|c|c|c|c|c|}
\hline UNIVERSIDADES/ PRO-REITORIAS & UFBA & UFS & UFAL & UFPE & UFRPE & UFPB & UFCG & UFRN & UFC & UFPI & UFMA & UFSB & UFOB & UFRB & UNIVASF & UFERSA & UFCA & UNILAB \\
\hline \multicolumn{19}{|l|}{ ASSISTENNCIA ESTIDANTIL } \\
\hline \multicolumn{19}{|c|}{ Açōes Afirmativas e Assistência Estudantit } \\
\hline \multicolumn{19}{|c|}{\begin{tabular}{|r|} 
Assuntos Comunitários \\
\end{tabular}} \\
\hline \multicolumn{19}{|c|}{\begin{tabular}{|l|l} 
AÇÕES AFIRIIATIVAS \\
\end{tabular}} \\
\hline \multicolumn{19}{|c|}{ Açoes Afirmativas e Assistência Estudantiti } \\
\hline \multicolumn{19}{|c|}{ Graduacào e Acōes Afirmativas } \\
\hline \multicolumn{19}{|c|}{ SUSTENTABILIDADE } \\
\hline \multicolumn{19}{|c|}{ Sustentabilidade e Integracão Social } \\
\hline \multicolumn{19}{|c|}{$\begin{array}{l}\text { TECNOLOGIA DA INFORIIAÇÃO E } \\
\text { CONINICACÃO }\end{array}$} \\
\hline \multicolumn{19}{|l|}{ RELACÕES INSTITUCIONAIS } \\
\hline CULTURA & & & & & & & & & & & & & & & & & & \\
\hline
\end{tabular}

Fonte: Autores, (2106). 


\section{$\checkmark \quad$ Assistência Estudantil}

Apesar de ser decorrente de uma política relativamente recente, o PNAES, a assistência estudantil já se encontra bem incorporada na forma como as universidades se departamentalizam. Como visto na tabela, ainda que possa haver mais de uma denominação (Assuntos ou Assistência), há pró-reitorias específicas para essa função em 16 universidades. Incluindo a UFCG, na qual a assistência estudantil é tratada pela Pró-Reitoria de Assuntos Comunitários. Já na UFOB o tema é abordado pela Pró-Reitoria de Graduação e Ações Afirmativas e na UFSB é tratado pela PróReitoria de Sustentabilidade e Integração Social.

\section{$\checkmark$ Ações Afirmativas}

Enquanto a Assistência Estudantil se encontra bastante consolidada, o mesmo não se observa em relação às ações afirmativas. Na UFBA, na UFRB e na UNILAB este tema é tratado pela Pró-Reitoria de Ações Afirmativas e Assistência Estudantil. E na UFOB há a Pró-Reitoria de Graduação e Ações Afirmativas. Dessa forma, ainda que possa haver algum outro órgão que trate do tema, numa instância hierarquicamente inferior, em apenas 4 universidades este tem o status de próreitoria. Sendo que três delas estão na Bahia, estado de maioria negra, e a UNILAB, na sua essência, foi concebida focando a cooperação solidária com os países de língua portuguesa, com destaque para os africanos.

\section{$\checkmark$ Tecnologia de Informação e Comunicação}

Em 3 universidades a Tecnologia de Informação e Comunicação é tratada numa pró-reitorias específica: UFSB, UFPE e UFOB.

\section{$\checkmark$ Outras}

Como casos diferenciados em relação ao conjunto das 18 universidades, um dos exemplos é a Pró-Reitoria de Sustentabilidade e Integração Social na UFSB, responsável pela formulação e execução de programas e projetos de sustentabilidade e também por as suntos comunitários e estudantis. Foi o único caso onde o tema da sustentabilidade recebeu esse destaque. Na UFCA há a Pró-Reitoria de Cultura, único caso onde a mesma recebe esse status exclusivo (ainda que haja 5 universidades onde Cultura está junto com Extensão). E na UNILAB existe a Pró-Reitoria de Relações Institucionais, que tem como objetivos promover e implementar a cooperação solidária com os outros países de língua portuguesa, com foco no sistema de mobilidade interinstitucional.

\section{CONCLUSÕES}

Considerando apenas o seu nível estratégico, composto pela Reitoria e pró-reitorias, foi possível perceber que neste nível predomina a departamentalização funcional, que tem por característica o agrupamento de funções ou atividades semelhantes. Além das 3 atividades primordiais das universidades, o Ensino/Graduação, a Pesquis a (geralmente junto com a Pós-Graduação) e a Extensão, na maioria há outras duas pró-reitorias dedicadas às atividades administrativas e uma à assistência estudantil. Esse padrão é típico tanto para as mais antigas quanto as novas universidades.

Nesse sentido, poucas inovações foram identificadas. Destaca-se a UFSB como um "ponto fora da curva", não apenas pelo número menor de pró-reitorias (4, quando a média geral está entre 6 e 7) mas pela forma como se organizou: Sustentabilidade e Integração Social, Planejamento e Administração, Gestão Acadêmica e Tecnologia de Informação e Comunicação. Das 7 novas universidades pesquisadas, em 6 delas a estrutura é muito similar às das tradicionais, o que indica um isomorfismo mimético entre elas. Considerando que os docentes das novas devem ter sido formados, em grande parte, nas já tradicionais, é de se esperar que se tenham adotando as formas das outras organizações.

Mesmo a incorporação da assistência estudantil na hierarquia das universidades aconteceu de forma similar entre as novas e as tradicionais. Diferenças pontuais foram observadas no conjunto, mas no geral, ainda que a criação de nova universidades representasse uma oportunidade à inovação, predominaram as "forças isomórficas".

\section{REFERÊNCIAS}

[1] BRASIL. MINISTÉRIO DA EDUCAÇÃO. Análise sobre a expansão das Universidades Federais 2003 a 2012. Brasília: MEC, 2012b.

[2] BRASIL. Lei ${ }^{\circ} 12.711$ de 29 de Agosto de 2012, Dispõe sobre o ingresso nas universidades federais e nas instituições federais de ensino técnico de nível médio e dá outras providências. Diário Oficial [da] Republica Federativa do Brasil, Brasília, 30 ago.2012a.

[3] BRASIL. Decreto No6.096, de 24 de abril de 2007, Dispõe sobre o Programa Nacional de Assistência Estudantil PNAES. Diário Oficial [da] Republica Federativa do Brasil, Brasília, 20 jul.2010.

[4] Lakatos, E.M.; Marconi, M.A. Fundamentos da Metodologia Científica. São Paulo: Atlas, 2003.

[5] Gil, A.C. Como elaborar projetos de pesquisa. São Paulo: Atlas, 2008.

[6] Lüdke, M.; André, M.E.D.A. Pesquisa em educação: abordagens qualitativas. São Paulo: EPU, 1986.

[7] Barros, A.J.S.; Lehfeld, N.A.S. Fundamentos de Metodologia Científica. São Paulo: Prentice-Hall, 2007.

[8] Yin, Robert. K. Estudo de caso: planejamento e método. 2. ed. São Paulo: Bookman, 2015.

[9] Triviños ANS. Introdução à pesquisa em Ciências Sociais: a pesquisa qualitativa em Educação. São Paulo, Atlas, 1987.

[10] Maximiniano, A.C.A. Introdução à administração. São Paulo: Atlas, 2000. 
[11] Dimaggio, P.J.; Powell, W.W. A gaiola de ferro revisitada: isomorfismo institucional e racionalidade coletiva nos campos organizacionais. Revista de Administração de Empresas, v.45, n.2, p.74-89, 2005.

[12] Redaelli, E. J. (2011). Isomorfismo como vantagem competitiva: um estudo de caso numa rede de distribuidores. Anais XIV Simpósio de Administração da Produção, Logística e Operações Internacionais, v. 1.

[13] Redaelli, E.J. Isomorfismo como vantagem competitiva: um estudo de caso numa rede de distribuidores. In: Simpósio de Administração da Produção, Logística e Operações Internacionais. XIV, 2011, São Paulo. Anais... São Paulo, 2011.

[14] Chiavenato, I. Introdução à Teoria Geral da Administração: uma visão abrangente da moderna administração das organizações. Rio de Janeiro: Elsevier, 2003.

[15] Oliveira, D.P.R. Sistemas, organizações e métodos: uma abordagem gerencial. São Paulo: Atlas, 2005.

[16] Kwasnicka, E.L. Introdução à administração. São Paulo: Atlas, 2006.

[17] Manganote, E.J.T. Organização, Sistemas \& Métodos. São Paulo: Alínea, 2001.

[18] Trigueiro, F.M.C.; Marques, N.A. Teorias da Administração I. Florianópolis: Capes, 2009. 